\title{
Analysis of expression of TNF-alpha and TGF-beta3 in intrinsic ureteropelvic junction obstruction
}

\author{
Koca $\mathrm{O}^{1}$, Kaya $\mathrm{C}^{1}$, Ozturk $\mathrm{MI}^{1}$, Gunes $\mathrm{M}^{1}$, Gumrukcu $\mathrm{G}^{2}$, Karaman $\mathrm{MI}^{1}$ \\ Department of Urology, Haydarpasa Numune Training and Research Hospital, Istanbul, Turkey. \\ drorhankoca@hotmail.com
}

\begin{abstract}
Objectives: Ureteropelvic junction (UPJ) obstruction is of critical importance to understand the histopathology of UPJ obstruction in terms of therapy planning and follow-up. For this purpose, our study was conducted with TNF- $\alpha$ and TGF- $\beta$ markers to investigate possible underlying problems in intrinsic UPJ obstruction. Methods: Of the patients who had undergone surgery in our clinic, 36 UPJ segments of patients who had undergone dismembered pyeloplasty surgery due to UPJ obstruction and 14 UPJ segments of the patients who had undergone nephrectomy were collected to form 2 groups. All histological sections were examined by applying immunohistochemical transforming growth factor beta 3 (TGF- $\beta 3$ ) and tumour necrosis factor alpha (TNF- $\alpha$ ) monoclonal antibody dyes.

Results: The mean staining values for TNF- $\alpha$ in mucosal tissue and mucosa were $0.53 \pm 0.84$ and $0.58 \pm 0.84$, respectively in the obstruction group, whereas the values observed in the control group were $0.86 \pm 0.36$ and $0.93 \pm 0.47$, respectively. While the mean staining values in the obstruction group in mucosal tissue and mucosa for TGF- $\beta 3$ were $1.75 \pm 0.73$ and $2.17 \pm 0.77$, respectively, the values established in the control group were $1.14 \pm 0.66$ and $1.43 \pm 0.93$, respectively. The difference between the obstruction and control groups were statistically significant for both values $(p<0.05)$.

Conclusion: Only a limited number of studies have been carried out on this particular issue. Data from the present study indicate that TGF- $\beta 3$ and TNF- $\alpha$ may play a role in the histopathogenesis of UPJ obstruction (Tab.

1, Fig. 1, Ref. 18). Full Text in PDF www.elis.sk.

Key words: ureteropelvic junction obstruction, TGF- $\beta 3$, TNF- $\alpha$, cytokine.
\end{abstract}

Ureteropelvic junction (UPJ) obstruction is the most common cause of hydronephrosis in children (1). Primary UPJ obstruction may be due to intrinsic proximal ureteral abnormalities, renal malrotation, ureteral kinks, and compression of the lower pole crossing renal vessels.

The etiopathogenesis of intrinsic UPJ obstruction is not clear, although it has been investigated embryologically, anatomically, functionally, and histologically (2). The defect that has commonly been reported was the presence of a non-peristaltic segment of ureter (2). Histopathologic studies have revealed such patients to have abnormal longitudinal muscular fibres or fibrous tissue instead of spiral muscles (2). Kaya et al showed an overexpression of extracellular matrix proteins and decreased nerve supply in intrinsic UPJ obstruction (3). Cutroneo et al demonstrated an increasing activation of apoptosis and a critical alteration in UPJ obstruction (4).

Cytokines are cellular regulatory proteins released by particular cells in response to various stimuli, which modify the behavior of targeted cells. The transforming growth factor beta (TGF- $\beta$ )

${ }^{1}$ Department of Urology, Haydarpasa Numune Training and Research Hospital, Istanbul, Turkey, and ${ }^{2}$ Department of Pathology, Haydarpasa Numune Training and Research Hospital, Istanbul, Turkey

Address for correspondence: O. Koca, MD, Haydarpaşa Numune Eğitim ve Araştırma Hastanesi II. Üroloji K1, Üsküdar, Istanbul, Turkey.

Phone: +90.216.4144502, Fax: +90 2163455982 family is comprised of a large group of extracellular growth factors, which have multiple effects on growth. TGF- $\beta$ family members consists of a large number of structurally similar polypeptide growth factors, which can arrange cellular processes, such as cell proliferation, differentiation, motility, adhesion, and death. TGF- $\beta$ induces fibrosis and an increased expression of TGF- $\beta 3$ can be related to fibrosis in UPJ. Tumour necrosis factor alpha (TNF- $\alpha$ ) is released from distal tubules and increases tubulointerstitial infiltration. The complexity of the immune system is regulated by cytokines and this is an interaction. They can influence both the humoral and cellular mechanisms, and stimulate the expression of adhesion molecules and the secretion of substances by macrophages (5).

Only a limited number of studies have been carried out on the histopathology of intrinsic UPJ obstruction. Knowing the etiopathogenesis of intrinsic UPJ obstruction is important for the treatment and follow up. For this purpose, our study was conducted with TNF- $\alpha$ and TGF- $\beta$ to investigate possible underlying problems in intrinsic UPJ obstruction.

\section{Patients and methods}

\section{Patient selection}

Of the patients who had undergone surgery in our clinic, 36 UPJ segments of patients who underwent dismembered pyeloplasty surgery due to UPJ obstruction (group 1) and 14 UPJ segments 
Tab. 1. TNF- $\alpha$ and TGF- $\beta 3$ muscular and mucosal staining in both groups and comparison of the groups.

\begin{tabular}{|c|c|c|c|c|}
\hline Obstruction & TNF- $\alpha$ Muscular & TNF- $\alpha$ Epithelium & TGF- $\beta 3$ Muscular & TGF- $\beta 3$ Epithelium \\
\hline Without obstruction $(\mathrm{n}=14)($ Mean \pm SD) & $0.86 \pm 0.363$ & $0.93 \pm 0.475$ & $1.14 \pm 0.663$ & $1.43 \pm 0.938$ \\
\hline With obstruction $(n=36)($ Mean \pm SD $)$ & $0.53 \pm 0.845$ & $0.58 \pm 0.841$ & $1.75 \pm 0.732$ & $2.17 \pm 0.775$ \\
\hline Total $(n=50)($ Mean \pm SD $)$ & $0.62 \pm 0.753$ & $0.68 \pm 0.768$ & $01.58 \pm 0.758$ & $1.96 \pm 0.880$ \\
\hline $\mathrm{P}$ & 0.021 & 0.036 & 0.007 & 0.009 \\
\hline
\end{tabular}

TGF- $\beta 3$ - Transforming growth factor-beta 3; TNF- $\alpha$ - Tumour necrosis factor-alpha; SD - Standard deviation

of patients (age range, 22-66 years) who underwent nephrectomy (without pelvis renalis tumours) (group 2) were collected to form 2 groups. There were 15 and 5 female patients in the Group 1 and 2 , respectively.

Obstruction cases of an extrinsic nature, such as aberrant vessel compression and intensive periureteral fibrosis were excluded from the study. Diagnosis of intrinsic UPJ obstruction was confirmed for all patients through radiologic and peri-operative findings. The indications for pyeloplasty included a $\mathrm{T}^{1} / 2$ value $>20$ minutes on diuretic scintigraphy, progressive deterioration in renal function, and symptomatic patients. Tissue $(2-3 \mathrm{~cm})$ from the UPJ region containing the UPJ was resected from control and UPJ obstruction cases. Solari et al. first identified the site where the funnel-shaped renal pelvis became continuous with the ureter and the resected 2-3 $\mathrm{mm}$ segment of the UPJ (6). All histological sections obtained from UPJs were examined immunohistochemically by using TGF- $\beta 3$ and TNF- $\alpha$ monoclonal antibody dyes.

\section{Immunohistochemistry}

Sections of 3-5 $\mu \mathrm{m}$ thickness from selected paraffin blocks were placed on positively-charged slides. The sections were kept at $56^{\circ} \mathrm{C}$ for 1 hour. The sections were incubated 3 xylene series for 10 minutes each. The sections were then dexylenized by incubating 3 alcohol series for 10 minutes each, and washed for 10 minutes in distilled water to be dealcoholized. Afterwards, the sections underwent antigen retrieval by boiling from a higher temperature to a lower temperature in citrate buffer in a microwave oven 4 times for 5 minutes at a time (for TNF- $\alpha, 10+5+5+10$ minutes). The sections were left in the same buffer for 20 minutes at room temperature to cool down and after being washed for 5 minutes with distilled water, the tissues were marked using a PAP-PEN. To eliminate endogenous peroxidase activation, $3 \%$ hydrogen peroxide solution (Peroxide Block, ACA008; ScyTek) was dropped and waited for 10 minutes. The sections were washed in phosphate buffer solution (PBS) and blocked by protein for 5 minutes (Super Block Ultra V, AA008; ScyTek). The sections were immersed in PBS and removed. Drops of TGF- $\beta 3$ (GTX15538 RTD readyto-use rabbit polyclonal antibody; Gene Tex, Inc.) and TNF- $\alpha$ (GTX26671 concentrated rabbit polyclonal antibody $(5 \mathrm{mg} / \mathrm{ml})$; Gene Tex, Inc.) were then added to the sections. The sections were incubated at room temperature for 1 hour for TGF- $\beta 3$ and 2.5 hours for TNF- $\alpha$, then washed in PBS for 10 minutes. A secondary antibody (UltraTek anti-polyvalent biotinylated secondary antibody, ABN008; ScyTek) was applied for 20 minutes, and the sections were washed in PBS for 10 minutes. The sections were then incubated for 20 minutes in a tertiary antibody (UltraTek HRP [Streptavidin], ABL008; ScyTek), and washed in PBS for 10 minutes. AEC chromagen (AEC chromagen and substrate buffer, ACD002-ACE005; ScyTek) drops were applied and the sections were kept at room temperature for 15 minutes. The sections were then washed in distilled water for 10 minutes. The tissues were contrast-stained in Mayer's hematoxylin (HMM008; ScyTek) for 1-2 minutes, and washed in distilled water for 2 minutes. The sections were mounted by using an aqueous mount.

\section{Evaluation of immunohistochemical staining}

Immunohistochemical examination revealed TNF- $\alpha$ and TGF- $\beta 3$ cytoplasmic staining. Stained cells were observed in the epithelium and muscular layer. Staining was scored as follows: no staining, 0; weak staining, 1) moderate staining, 2) and intense staining, 3) the numerical values of the cells with positive staining were established with respect to the area with the most intense staining. Positive controls for TNF- $\alpha$ and TGF- $\beta 3$ staining were carried out using tonsils and placentas, respectively. All specimens were examined under light microscope by the same observer (G.G).

The segments of the intrinsic UPJ obstruction group were compared to the segments from the normal UPJ group. Statistical analyses were carried out using the Mann-Whitney U test. Statistical significance level was considered as $\mathrm{p}<0.05$.

\section{Results}

The mean age of the patients was $27.7 \pm 18.43$ (3-66) years in the intrinsic UPJ obstruction group (Group 1), while it was $51.7 \pm 15.66$ (22-66) years in the normal UPJ group (Group 2) $(\mathrm{p}<0.05)$.

HE staining revealed that all patients with intrinsic UPJ obstruction ( $\mathrm{n}=36)$ had lumen stenoses, thickening accompanied by fibrosis in the lamina propria, and elevated amounts of connective tissue among muscle fibres in the muscularis propria in all regions of obstruction (100\%) when compared to normal findings; however, the findings were not present in the proximal or distal surgical margins, or was at a minimal level. Of the patients, $6(16.66 \%)$ were observed to have mild or increased pathologic obstruction region characteristics in the distal surgical margin.

In the obstruction group, the TNF- $\alpha$ staining values in the muscular tissues and epithelium were $0.53 \pm 0.84$ and $0.58 \pm 0.84$, respectively, while the staining values for the controls were $0.86 \pm 0.36$ and $0.93 \pm 0.47$, respectively (Tab. 1). The difference between the obstruction group and the controls for both values was statistically significant $(\mathrm{p}<0.05)$ (Tab. 1, Figs 1a, 1b).

In the obstruction group, the TGF- $\beta 3$ staining values in the muscular tissues and epithelium were $1.75 \pm 0.73$ and $2.17 \pm 0.77$, respectively, while the staining values for the controls were 


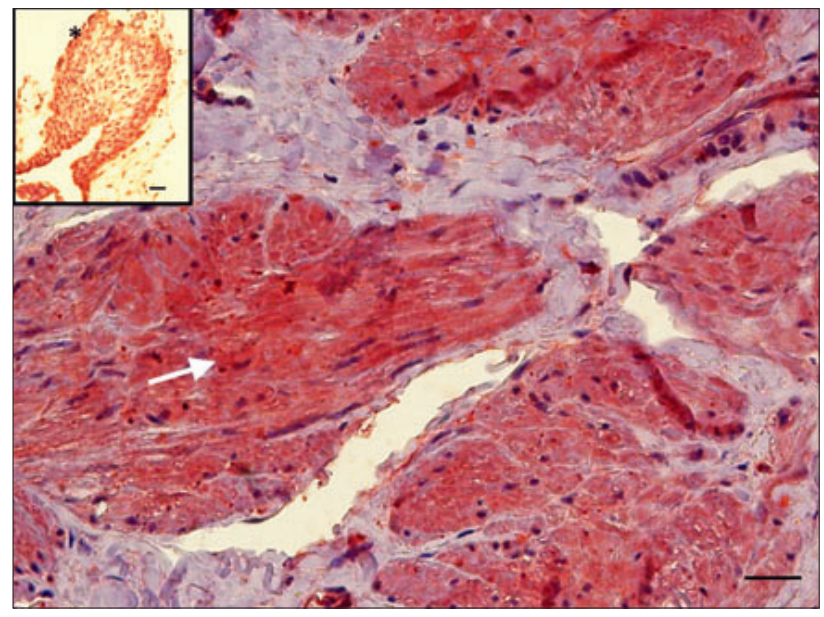

Fig. 1a. Intensive staining in cytoplasm of smooth muscle cells with TNF- $\alpha$ (arrow), epithelial staining with TNF- $\alpha(*)$; original magnification: $x 400$, small picture: x100, scale-bar: $100 \mu \mathrm{m}$.

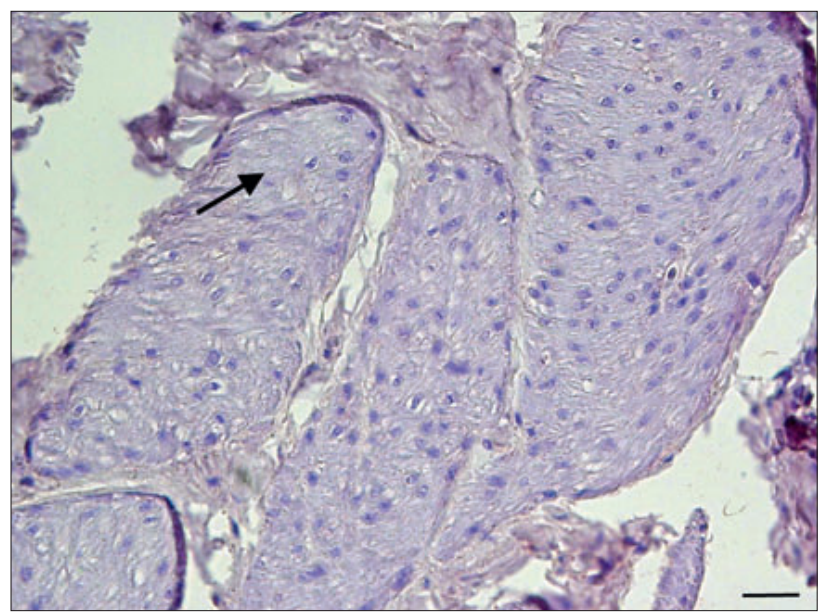

Fig. 1b. Negative staining of UPJ with TNF- $\alpha$ (x400), scale-bar: $100 \mu \mathrm{m}$.

$1.14 \pm 0.66$ and $1.43 \pm 0.93$, respectively (Tab. 1). The difference between the obstruction group and the controls for both values was statistically significant $(\mathrm{p}<0.05)$ (Tab. 1, Figs $1 \mathrm{c}, 1 \mathrm{~d})$.

When the patients $<30$ years of age and $>30$ years of age were divided into 2 groups, it was observed that TNF- $\alpha$ staining was lower in the patients $<30$ years of age. The difference between the groups was statistically significant $(\mathrm{p}<0.05)$. The change with respect to age was not significant for TGF- $\beta 3$ staining $(p>0.05)$. In the case of age groups, there was not any statistical difference between the values of pediatric and adult patients compared to normal cases $(\mathrm{p}>0.05)$.

\section{Discussion}

Although UPJ obstruction can occur in all age groups, it is the most common cause of neonatal hydronephrosis (7). Although the diagnosis is made in $25 \%$ of the cases at $0-1$ years of age, it can often be diagnosed prenatally (8). The aetiology of intrinsic

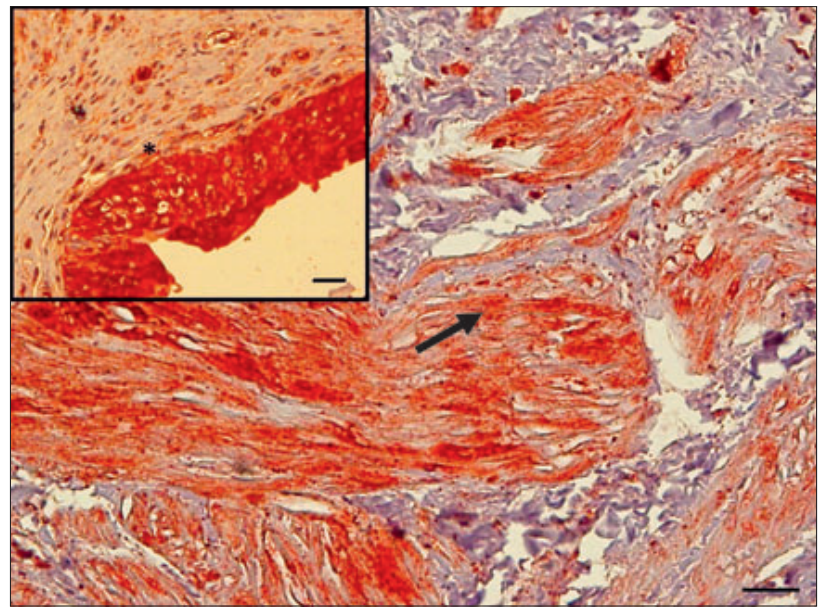

Fig. 1c. Intensive staining in cytoplasm of smooth muscle cells with TGF- $\beta 3$ (arrow), epithelial staining with TGF- $\beta 3(*)$; original magnification: x400, small picture: x200, scale-bar: $100 \mu \mathrm{m}$.

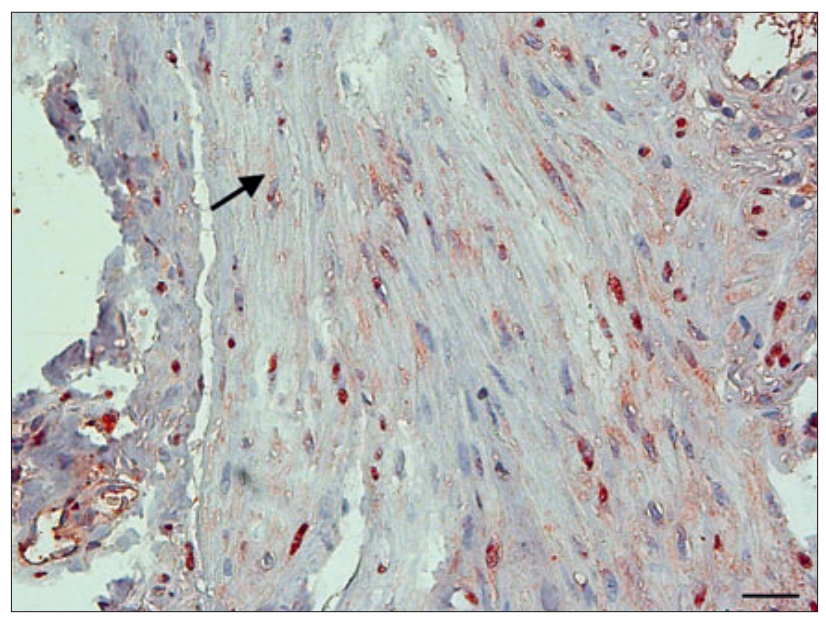

Fig. 1d. Weak staining of UPJ with TGF-ß3 (x400), scale-bar: $100 \mu \mathrm{m}$.

UPJ obstruction has not been clearly elucidated. Histopathologic studies have investigated UPJ obstruction in a wide range. Detailed documentation of pathologic segments in UPJ obstruction has been made by Pinter et al. with classification into five groups (from the normal UPJ (almost) lumen to no lumen with thickened wall which infiltrates with intensive collagen) (9).

The success rate of dismembered pyeloplasties in the surgical management of intrinsic UPJ obstruction has been reported to be $93-100 \%(10,11)$. The mean age of patients undergoing surgery due to UPJ obstruction was 27.67 years, which is higher than the values reported in literature (7). A multicenter study conducted in Turkey indicated the mean age of admission to doctor due to UPJ obstruction to be 22 years (12). By improving the opportunities for antenatal diagnosis (by ultrasound), surgery can be performed for patients in Turkey who are in need for surgery at a younger age, increasing the chances of preventing irreversible renal damage.

It does not seem likely that the cause of UPJ obstruction can be explained with a single theory. Murakumo et al. emphasized 
the importance of a multifaceted approach combining neurogenic and myogenic theories in understanding the etiopathogenesis of intrinsic UPJ obstruction (13). A recent non-invasive study, which focused on the measuring urinary TGF- $\beta 1$ in children with congenital UPJ obstruction confirmed this urinary cytokine as a useful tool for the diagnosis and follow-up of UPJ obstruction (14).

TNF- $\alpha$ stimulates mesangial cell contraction and proliferation, and increases the synthesis and secretion of chemokine and lipid mediators in renal cells. TNF- $\alpha$ leads to elevated cell apoptosis as well. TNF- $\alpha$ achieves this by decreasing mRNA levels of gen bcl-2, which inhibits apoptosis while increasing gen Fas expression, which stimulates apoptosis in target cells (15). TNF- $\alpha$ was observed with negative staining in muscular tissues and urothelium in the UPJ obstruction group in the present study, suggesting an impaired functioning of these regulators during prenatal and postnatal development. Similarly, the lower staining rates in older patients suggested that the effectiveness of TNF- $\alpha$ changed over time during the developmental process. Although the mean age was higher in the control group when compared to the study group, staining was more intense. Therefore, if the present study is repeated with age-matched groups, the difference in staining could be even more significant.

The TGF- $\beta$ family is comprised of a large group of extracellular growth factors, which control the development with multiple effects. TNF- $\beta$ is released from proximal tubules and stimulates extracellular matrix composition. Platelet-derived growth factor is released from tubular cells and stimulates renal fibroblast production under in vitro conditions (16). Alterations have been established in TGF- $\beta$ signal pathway in a number of disorders. Impaired transmission of the growth inhibitor response to TGF- $\beta$ is a common condition observed in cancer cells. Elevated TGF- $\beta$ activity plays a central role in fibrotic diseases in the lungs, kidneys, liver, and other organs characterized by increased matrix material in tissues (17). TGB- $\beta 3$ was implicated as a likely cause of fibrosis leading to obstruction due to the significantly more intense staining observed in the UPJ obstruction group when compared to the control group in our study. However, in literature, TGF- $\beta 3$, in contrast to TGF- $\beta 1$ and TGF- $\beta 2$, is generally assumed to have anti-inflammatory, anti-fibrotic, and anti-apoptotic properties. This view is supported by the fact that control of mesenchymal cell proliferation and differentiation, and extracellular matrix production are among the functions of the TGF- $\beta$ family. Change in staining with TGF- $\beta 3$ with respect to age does not demonstrate significant difference, however, a study carried out with age-matched groups may reveal even more significant results, despite the fact that the different mean ages of the groups do not appear to have had a negative impact on the staining results.

The urothelium is a metabolically active tissue responding to chemical and mechanical stimuli as well as interacting chemically with the nerves on bladder walls. It has strong physical connections with afferent nerves and triggers local vascular alterations and/or bladder contractions by releasing ATP and NO (18). In our study, we observed the effect of urethelium pathologies on the UPJ as well. Significant differences between the obstruction and control groups in terms of staining observed with both TGF- $\beta 3$ and TNF- $\alpha$ in muscular and epithelial tissue suggested that certain mediators released or failed to be released from muscular tissue in parallel to epithelial tissues may be implicated in the histopathology of UPJ obstruction. The different staining rates of muscular and epithelial tissues in our study may be caused by the different response capacities of these tissues. However, further studies with larger study samples should be conducted on this particular issue in order to confirm the existence of this particular relation.

The weak point of our study is that the mean age in the obstruction and control group in the present study was higher than those reported in literature for patients with UPJ obstruction. Unable to enrol patients consistent with the mean age of patients having particular therapy is one of the constraints of the present histopathologic study. The number of pediatric patients in both groups was remarkably low. In order to investigate the histopathology of such cases, UPJ segments should be obtained from subjects within a suitable age group both for the study and control groups. However, particularly for the control group, it is rather difficult to obtain samples. Healthy UPJ segments can only be obtained from cadavers or patients who have undergone nephrectomies. As cadaver studies cannot be carried out in our country for socio-cultural reasons and legal obstacles, it is practically impossible to obtain healthy UPJ segments from the younger age groups. However, we thought that studies should be conducted with sufficiently large study samples in order to reach valid histopathologic conclusions.

The histopathology of intrinsic UPJ obstruction has not been understood clearly. We believe that understanding the histopathology of these congenital diseases will lead the way to the use of the agonists or antagonists of certain mediators in the neonatal period, offering non-surgical therapy choices, and it will also play a critical role in evaluating therapeutic alternatives, and in establishing post-operative or non-surgical follow-up criteria.

\section{References}

1. Wang Y, Puri P, Hassan J, Miyakita H, Reen DJ. Abnormal innervation and altered nerve growth factor messenger ribonucleic acid expression in ureteropelvic junction obstruction. J Urol 1995; 154: 679-683, PMID: 7609154.

2. Gasling JA, Dixon JS. Functional obstruction of the ureter and renal pelvis: A histological and electron microscopic study. Br J Urol 1978; 50 : 145-152, PMID: 753449

3. Kaya C, Bogaert G, de Ridder D, Schwentner C, Fritsch H, Oswald J, Radmayr C. Extracellular matrix degradation and reduced neural density in children with intrinsic ureteropelvic junction obstruction. Urology 2010; 76: 185-189, PMID: 20303154.

4. Cutroneo G, Arena S, Anastasi G, Cervellione RM, Grimaldi S, Di Mauro D, Speciale F, Arena F, Di Benedetto V, Favaloro A, Magno C. Altered cytoskeletal structure of smooth muscle cells in ureteropelvic junction obstruction. J Urol 2011; 185: 2314-2319, PMID: 21511284.

5. Güneş H. Effects of cytokines on cell cycle. Tr J Biol 1999; 23: 283-292.

6. Solari V, Piotrowska AP, Puri P. Altered expression of interstitial cells of Cajal in congenital ureteropelvic junction obstruction. J Urol 2003; 170: 2420-2422, PMID: 14634443. 
498-502

7. Barry AK. Ureter and uretropelvic junction disease. In: Smith's General Urology. Edited by Mc graw- Hill. 2000, pp: 628-641.

8. Carr MC, El-Ghoneimi A. Anomalies and surgery of the ureteropelvic junction in children. Campbell-Walsh urology. 9th ed. Saunders Elsevier; Philadelphia, 2007. pp. 3359.

9. Pinter AB, Horvath A, Hrabovszky Z. The relationship of smooth muscle damage to age, severity of pre-operative hydronephrosis and postoperative outcome in obstructive uropathies. Br J Urol 1997; 80: 227-233, PMID: 9284193.

10. Harish J, Joshi K, Rao KL. Pelviureteric junction obstruction: how much is the extent of the upper ureter with defective innervation needing resection? J Pediatr Surg 2003; 38: 1194, PMID: 12891491.

11. Weiss RM. Physiology and pharmacology of the renal pelvis and ureter. Campbell-Walsh urology. 9th ed. Saunders Elsevier; Philadelphia, 2007. pp. 1910-1916.

12. Karaman MI, Ozturk M, Oktar T, Müslümanoğlu AY, Güney S, Polat H, Cangüven Ö. Ureteropelvic junction stenosis: A multicenter study in terms of patient profile and surgical approach. (Abstract) Turkish Journal of Urology, Congress special issue, 2006, p. 314.
13. Murakumo M, Nonomura K, Yamashita T. Structural changes of collagen components and diminution of nerves in congenital ureteropelvic junction obstruction. J Urol 1997; 157: 1963, PMID: 9112572.

14. Sager C, Lopez JC, Duran V, Burek C, Perazzo E. Transforming growth factor-beta1 in congenital ureteropelvic junction obstruction: diagnosis and follow-up. Int Braz J Urol 2009; 35: 315-323, PMID: 19538767.

15. Abboud HE. Growth factors in glomerulonephritis. Kidney Int 1993; 43: 252-267, PMID: 8433565.

16. Matrisian LM. Metalloproteinases and their inhibitors in matrix remodelling. Trends in Genetics 1990; 6: 121-125, PMID: 2132731.

17. Kalman S. Cytokins and growth factors in renal injury. Official Journal of the Turkish Society of Nephrology 2002; 11: 140-143.

18. de Groat WC. The urothelium in overactive bladder: passive bystander or active participant? Urology 2004; 64: 7-11, PMID: 15621221.

Received August 16, 2011. Accepted January 17, 2012. 